\title{
SIGNIFICANCE OF FLEXING MINIMUS WITHOUT RING FINGER- IN TAMILNADU POPULATION
}

\section{J. Chandrapooja ${ }^{1^{*}}$ \& Saravana Kumar ${ }^{2}$}

\author{
${ }^{1 *}$ Saveetha Dental College, Poonamalle High Road, Velappanchavadi, Chennai 660077 \\ 2Depatment of Anatomy, Saveetha Dental College, 162, Poonamallee High Road, Chennai, Tamilnadu-600077
}

\begin{abstract}
AIM: The aim is to evaluate the significance moving our little finger without the ring finger, along with it in, Tamilnadu population. BACKGROUND: Most of the people cannot flex their minimus, without the rind finger, along with it, this occurs because of human anatomy. The muscle connected to the tendons for those fingers are close or connected to the same. The tendons and bones are usually connected for last two fingers. For most people it weakens as they grow, but it's just a gene in they are family to have stronger connections of those tendons. [1] Other than arrector pili in the skin, fingers do not contain muscles. The flexor muscles, which are used to bend the fingers, are located in the palm and forearm. The muscle bulks that move each finger may be partly blended, and the tendons may be attached to each other by a net of fibrous tissue, preventing completely free movement. The extent of this can differ from person to person [2]. REASON: This study was conducted in order to know the significance of flexing the minimus without ring finger.
\end{abstract}

\section{KEY WORDS}

Minimus [the little finger], Tendons, Gene.

\section{INTRODUCTION}

General finger flexion is controlled by three groups of muscles - flexor digitorum superficialis and flexor digitorum profundus and the lumbricals. Flexor digitorum profundus crosses all three finger joints and so, when acting alone, will flex all three joints simultaneously. Flexor digitorum superficialis crosses only the first two joints and so cannot flex the distal interphalangeal joint. The lumbricals arise from the tendons of the flexor digitorum profundus muscle but insert onto the common extensor sheath of the extensor digitorum muscle. When a lumbrical contracts in isolation it causes the finger to flex at the metacarpophalangeal joint and extend at the proximal and distal interphalangeal joints. [3] People have different abilities to control the independent movement of their fingers - some of these abilities are learned and some are natural - but they are basically the function of these three muscles. This interplay of muscle function basically explains how you can move your middle and ring fingers.

When you flex your fingers, bend them forward to form a fist or extend them, straighten them out the fingers they move at three joints. The metacarpophalangeal joint, next is the proximal interphalangeal joint and last is the distal interphalangeal joint are the joints closest to the hand. Different sets of muscles to control these joints in different ways. While extending the fingers, the extensor digitorum muscle, extend the fingers at all three joints at the same time. For flexing the fingers at only one joint we need to selectively overpower the extensor digitorum muscle. [3]

As the two tendons (muscle fibers) that raise the fingers (little finger and ring finger) both connect to the same lump of muscle in your forearm. Supplied by Ulnar Nerve, The ulnar nerve gives feeling to the little finger and half of the ring finger. It also controls most of the little muscles in the hand that help with fine 
movements. If entrapment or irritation to this UInar Nerve is the reason for your problem. [4]

The index finger and pinky are special cases. Both fingers have distinct and independent extensor muscles (extensor indicis and extensor digiti minimi). The action of these muscles allows you to fully extend your index finger or pinky while the other fingers are flexed.

The flexor digitorum muscles are power muscles, without much independent control. When you flex your ring finger you are using these flexor muscles, which to some extend are acting upon all the fingers simultaneously. In most people when they flex just the ring finger they are still producing some movement in the other fingers, especially the middle finger. The pinky won't move much, if at all, because it has the extensor digiti minimi muscle to keep it extended. The middle finger doesn't move much because it is a longer finger and the "slack" that forms in the extensor digitorum tendon by flexing one of the other fingers will not affect it as much. However, when you flex the pinky at the proximal interphalangeal joint you need to overcome the power of extensor digitorum to keep the other joints extended. Because the ring finger does not have an independent extensor muscle it must flex along with the pinky.

While recognising gross movements, like complex movements such as wrist and finger movements, a number of muscles are involved. [5]

There are some people that do have independent extensor muscles for the middle and ring fingers. Having one for the middle finger is fairly common about 1 in 20 people. Having an independent ring finger extensor is less common. However, if you can find someone who can keep their ring finger straight while they flex their pinky you will have found one of those people with the independent ring finger extensor muscle.

\section{MATERIALS AND METHODS}

People were asked to bend their pinky finger slightly first, and checked whether their ring finger is also getting bent. Then they were asked to try to bend only their pinky finger without flexing their ring finger. Some were able to do without straining, others tried to do it and the remaining was not able to it by any chance. Two columns were made name of the people who can bend their pinky without bending their ring finger were noted in one column and name of people who cannot do it were noted in another column. The sample size was 100 . The research was done in tamilnadu population. Then the results were interpreted in accordance with the norms.

\section{RESULT}

People who can flex mininus without 33
bending ring finger
people who cannot flex minimus without 67
bending ring finger

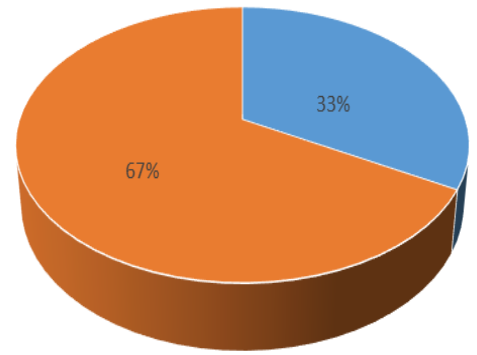

- People who can flex mininus without bending ring finger - people who cannot flex minimus without bending ring finger

\section{DISCUSSION}

When we analyse the results we come to know that $33 \%$ of people who can flex their pinky finger without bending their ring finger are mostly dancers or people try to bend and play with their fingers. Their muscles and tendons are free and flexible compared to others. The remaining people who cannot do this have stronger flexors in their fingers. The thumb, index, and middle finger all usually have separate flexor tendons. While the ring and little finger also typically have their own flexor tendons, it is very common to have some branching of one to the other. So when one flexes, the other is obliged to come along too. [6]

\section{CONCLUSION}

Many people have an anatomical variation of the forearm muscles where the muscle that extends the pinky is fused with the one that extends the other 
fingers of the hand. This makes it difficult for the independent operation of the pinky and ring fingers. [7] So this bending of pinkies without flexing ring finger does not play a great role in human anatomy, but it helps in certain things like playing certain instruments. It's a harmless variation of normal anatomy, like whether or not you're able to roll your tongue.

\section{REFERENCE}

[1] Why can't I move my ring fingers and pinkies independently from each other? Dayton Skelton, A person with a low IQ, Quora.

[2]Why can't most people move/bend individual fingers without moving any others? Mikaela Sifuentes, Ph.D. student in Neuroscience, Quora.
[3] If I bend my pinky, why does my ring finger bend too? Thomas M. Greiner, Assistant Professor of Anatomy, Area of science: Anatomy, ID: 1116309146.An

[4] Why can I not lift my right pinky finger upright? Legna Mahi, Quora.

[5] Childress DA. Proceedings of 8th International Conference on Medical and Biological Engineering conference (ICMBE) Chicago: Palmer House; 1969. A myoelectric three state controller using rate sensitivity; pp. 4-5

[6] Why can't I move my ring fingers and pinkies independently from each other? Peter J Ward, Writer in Anatomy. Quora.

[7] Why can't I move my ring fingers and pinkies independently from each other, John Wallace, Professional biologist with training in anatomy, physiology, and medical tech, Quora. 